\title{
Comer ou não comer, eis a questão: diferenças de gênero na neofobia alimentar
}

\author{
Fivia de Araújo Lopes ${ }^{1}$ \\ Juliana Severo Procópio Cabral \\ Luisa Helena Pinbeiro Spinelli \\ Luiza Cervenka \\ Maria Emilia Yamamoto \\ Rochele Castelo Branco \\ Wallisen Tadashi Hattori
}

\begin{abstract}
Resumo
A neofobia alimentar é um comportamento de cautela quanto à ingestão de alimentos desconhecidos. Homens e mulheres apresentam diferenças comportamentais, embora as pesquisas sobre a resposta neofóbica apontem controvérsias. Neste estudo, 266 indivíduos realizaram uma tarefa de escolha entre dois alimentos, um familiar e outro não-familiar, e responderam a uma escala de neofobia, a fim de investigar as diferenças de gênero. Os resultados indicaram que as mulheres são mais neofóbicas que os homens, o que corrobora os dados anteriores sobre o comportamento alimentar e pode estar relacionado ao papel feminino de seleção e preparo do alimento no âmbito familiar.
\end{abstract}

Palavras-chave: Neofobia alimentar; Comportamento alimentar; Diferença entre gêneros.

\section{Eating or not eating, that's the question: gender differences on food neophobia}

\begin{abstract}
Feeding neophobia represents a cautious behavior towards unknown food. Although men and women show behavioral differences, relating feeding neophobia data on neophobic response are controversial. In this study, in order to investigating gender differences, 266 individuals were tested on a two food choosing task between a familiar and an unfamiliar food items, and also were required to fill in a food neophobia scale. Results indicate that women are more neophobic than men confirming previous research data on feeding behavior. This might be related to females' role to selecting and preparing food in the family context.

Keywords: Food neophobia; Feeding behavior; Gender differences.
\end{abstract}

\section{Introdução}

A diversidade da dieta dos seres humanos é surpreendente, conferindo vantagens adaptativas, mas proporcionando a complexa tarefa de decidir o que devemos comer. Muitas vezes tal decisão envolve uma certa relutância em ingerir alimentos novos. Este comportamento é denominado neofobia alimentar, o qual proporciona uma função protetora em um ambiente de possíveis itens danosos ao organismo humano (Pliner \& Hobden, 1992; Yamamoto \& Lopes, 2004). No entanto, a resposta neofóbica não é estática, havendo uma tendência de diminuição com o avanço da idade (Birch, 1999; Hursti \& Sjödén, 1997) e a própria história de vida do indivíduo apresenta diversas variáveis que podem influenciar esse comportamento (Hursti \& Sjödén, 1997, Hendy \& Raudenbush, 2000, Rozin, 1996).

As pesquisas a respeito das diferenças entre gêneros quanto a neofobia alimentar apontam resultados controversos, sugerindo que o traço neofóbico é mais acentuado em homens (Koivisto \& Sjöden, 1996; Marcelino, Adam, Couronne, Köster \& Sieffermann, 2001), ou em mulheres (Ramezani \& Roeder, 1995). Outros estudos indicam ainda que esse traço não difere entre os sexos (Nordin, Broman, Garvill \& Nyroos, 2004). Partindo dessa controvérsia, o objetivo de nosso trabalho foi investigar possíveis diferenças entre os gêneros quanto à resposta neofóbica.

\section{Método}

Sujeitos

Nossa amostra consistiu de 266 indivíduos. Desse total, 109 eram homens com idade variando entre 15 e 57 anos $(\xi=23,38$ anos; d.p. $= \pm 9,83$ anos $)$

\footnotetext{
${ }^{1}$ Endereço para correspondência:

Universidade Federal do Rio Grande do Norte - Centro de Biociências - Departamento de Fisiologia

Caixa Postal 1.511 - Lagoa Nova - 59078-970 - Natal-RN

Telefone 84-3215-3409 - Fax 84-3211-9206 - E-mail: fivialopes@yahoo.com.br
} 
e 157 mulheres de 15 a 54 anos $(\xi=22,29$ anos; d.p. $=$ $\pm 7,80$ anos). A escolaridade dos participantes variou de ensino fundamental incompleto a ensino superior completo.

\section{Procedimento experimental}

Os sujeitos foram testados em um experimento consentido numa Feira de Ciências e Tecnologia (CIENTEC - UFRN), em novembro de 2004. Os voluntários foram submetidos individualmente a uma escolha entre dois tipos de pastas, uma familiar (patê de presunto ou de queijo com ervas) e outra não (guacamole - pasta mexicana - ou tapenade - pasta francesa). Foram incluídas na amostra as pessoas que conheciam apenas um dos dois alimentos apresentados. Após o teste, os indivíduos responderam à escala de neofobia alimentar proposta por Pliner \& Hobden (1992).

\section{Análise estatística}

Utilizamos o teste do Qui-quadrado para verificar diferenças entre os sexos e o teste $t$ de Student para investigar as diferenças de pontuação na escala de neofobia. O nível de significância adotado para todos os testes foi menor ou igual a 0,05 .

\section{Resultados}

Considerando o gênero, observamos que homens e mulheres não diferem na escolha geral, mas verificamos uma tendência do sexo masculino em se mostrar menos neofóbicos do que as mulheres $\left(\chi^{2}=\right.$ 3,53; g.1. $=1 ; \mathrm{p}=0,0603$ ) (Figura 1). Considerando apenas a escolha feminina, as mulheres preferem

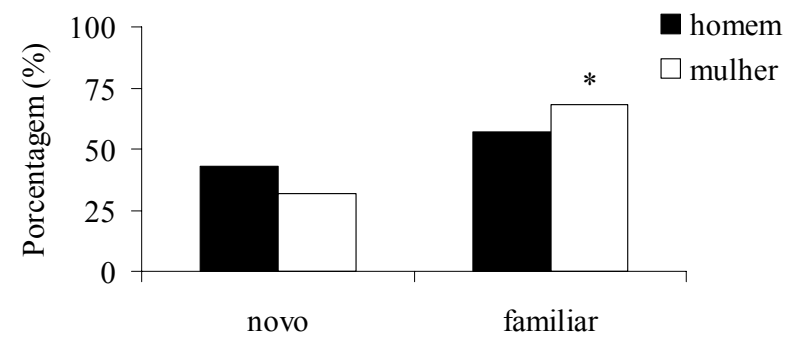

Tipos de alimento

Figura 1 - Porcentual da escolha entre alimento novo e familiar para homens e mulheres. * freqüência significativamente maior de consumo do alimento familiar comparado ao alimento novo, entre as mulheres. significativamente os alimentos conhecidos aos novos $\left(\chi^{2}=20,69 ;\right.$ g.l. $\left.=1 ; \mathrm{p}<0,01\right)$ (Figura 1$)$. Em relação à escala de neofobia, verificamos que as mulheres se apresentam significativamente mais neofóbicas do que os homens $(t=2,08 ; \mathrm{p}=0,03)$ (Figura 2).

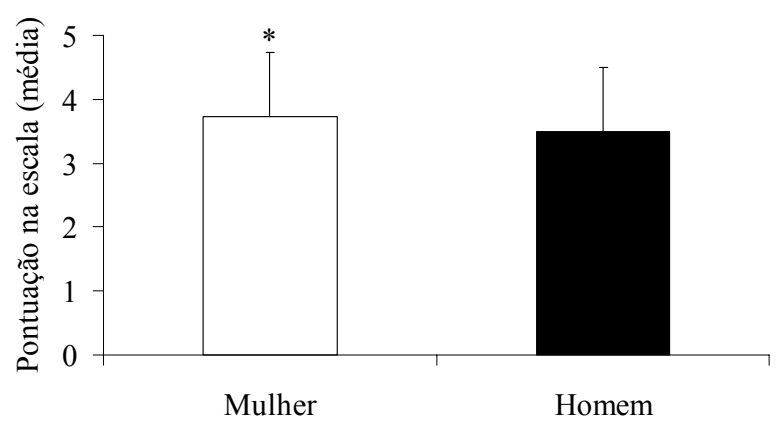

Figura 2 - Média e desvio padrão da pontuação da escala de neofobia alimentar (Pliner \& Hobden, 1992). * pontuação significativamente maior do que os homens.

\section{Discussão}

Diversos comportamentos humanos são dimórficos com relação ao gênero (Geary, 1998). No entanto, é importante observar que a maioria das diferenças comportamentais entre os sexos é mais uma questão de grau do que de tipo, ou seja, ambos apresentam um dado comportamento, mas em intensidades diferentes (Hines, 2004).

A literatura sobre o comportamento alimentar sugere diferenças entre homens e mulheres, com estas apresentando maior rejeição alimentar do que os homens (Mooney \& Walborurn, 2001; Nordin et al., 2004). Em nosso trabalho, a reposta de neofobia alimentar foi mais acentuada para as mulheres, tanto no que se refere à escolha propriamente dita, quanto às respostas na escala de neofobia. Rozin, Fischler, Imada, Sarubin \& Wrzesniewski (1999) sugerem que as mulheres são cautelosas quanto à escolha do alimento no que se refere à saúde, e que talvez isso esteja relacionado com a responsabilidade de seleção e preparo dos alimentos para as suas famílias.

\section{Referências}

Birch, L. L. (1999). Development of Food Preferences. Annual Review of Nutrition, 19, 41-62.

Geary, D. (1998). Male, female: the evolution of human sex differences. Washington, D.C.: American Psychological Association. 
Hendy, H. M. \& Raudenbush, B. (2000). Effectiveness of teacher modeling to encourage food acceptance in preschool children. Appetite, 34, 61-76.

Hines, M. (2004). Brain Gender. New York, NY: Oxford University Press.

Hursti, U.-K. K. \& Sjödén, P.-O. (1997). Food and general neophobia and their relationship with selfreported food choice: familiar resemblance in Swedish families with children of ages 7-17 years. Appetite, 29, 89-103.

Koivisto, U.-K. \& Sjöden, P.-O. (1996). Food and general neophobia in Swedish families: parent-child comparisons and relationships with serving specific foods. Appetite, 26, 107-118.

Marcelino, A. S., Adam, A. S., Couronne, T., Köster, E. P. \& Sieffermann, J. M. (2001). Internal and external determinants of eating initiation in humans. Appetite, 36, 914.

Mooney, K. M. \& Walbourn, L. (2001). When college students reject food: not just a matter of taste. Appetite, 36, 41-50.

Nordin, S., Broman, D. A., Garvill, J. \& Nyroos, M. Gender differences in factors affecting rejection of food in healthy young Swedish adults. Appetite, 43, 295-301.
Pliner, P. \& Hobden, K. (1992). Development of a scale to measure the trait of food neophobia in humans. Appetite, 19, 105-120.

Ramezani, C. A. \& Roeder, C. (1995). Health knowledge and nutritional adequacy of female heads of households in the United States. Journal of Consumer Affairs, 29, 381-402.

Rozin, P. (1996). Sociocultural influences on human food selection. Em E. D. Capaldi (Org.) Why we eat what we eat (pp. 233-262). Washington, DC: American Psychological Association.

Rozin, P, Fischler, C., Imada, S., Sarubin, A., \& Wrzesniewski, A. (1999). Attitudes to food and the role of food in life in the USA, Japan, Flemish Belgium and France: Possible implications for the diet-health debate. Appetite, 33, 163-180.

Yamamoto, M. E. \& Lopes, F. A. (2004). Dize-me o que falas e te direi o que comes: aquisição da linguagem e composição da dieta em crianças. Em M. L. S. Moura (Org.). O bebê do século XXI e a psicologia em desenvolvimento. São Paulo, SP: Casa do Psicólogo.

Recebido em setembro de 2005 Aprovado em junho de 2006

Sobre os autores:

Fívia de Araújo Lopes: psicóloga, doutora em Psicobiologia (UFRN) e professora adjunta do Departamento de Fisiologia, Centro de Biociências (UFRN).

Juliana Severo Procópio Cabral: psicóloga, mestranda do Programa de Pós-Graduação em Psicobiologia (UFRN).

Luísa Helena Pinheiro Spinelli: psicóloga, mestre em Psicobiologia (UFRN), doutoranda do Programa de PósGraduação em Psicobiologia (UFRN).

Luíza Cervenka: graduanda do curso de Ciências Biológicas (UFRN).

Maria Emilia Yamamoto: psicóloga, doutora em Psicofarmacologia (UNIFESP), professora titular do departamento de Fisiologia, Centro de Biociências (UFRN) e pesquisadora 1A do CNPq. Coordenadora do Projeto de Psicologia Evolucionista (CNPq - Institutos do Milênio).

Rochele Castelo Branco: bióloga, mestre em Psicobiologia (UFRN).

Wallisen Tadashi Hattori: biólogo, mestre em Psicobiologia (UFRN), doutorando do Programa de PósGraduação em Psicobiologia (UFRN). 
